\title{
Gram-Positive S. mitis Become Sensitive to Colistin and Nalidixic Acid when Grown in Xylitol
}

Gail Czarnecki², Prabani Dissanayake ${ }^{1}$ and Sunil Palchaudhuri ${ }^{*}$ *

${ }^{1}$ Department of Immunology and Microbiology, Wayne State University, School of Medicine, Detroit, MI, USA

${ }^{2}$ Department of Orthodontics and Pediatric Dentistry, University of Michigan, School of Dentistry, Ann Arbor, MI, USA

\begin{abstract}
The generation time of diplococcic viridans group streptococci, S. mitis is unusually prolonged when grown in rich broth containing xylitol ( $2 \%$ or higher concentrations). These xylitol-exposed streptococci predominantly grow in chains of varying lengths with heterogeneity of color: pink, purple and pink and purple combined. The pink portion of these bacterial chains appears to be thinner than the purple portion indicating a reduction in cell wall thickness. In support of optical microscopy, scanning electron microscopy of the same bacterial population demonstrates a considerable reduction of sizes but still in chains. In these chains, there is a variation in size indicating that all members of the diplococcic S. mitis population are not equally affected. Apparently, the partially affected viable population of $S$. mitis, becomes sensitive to colistin and nalidixic acid when grown in xylitol.
\end{abstract}

Keywords: Xylitol; Cell wall thickness; S. mitis; Colistin; Nalidixic acid; Electron microscopy

\section{Introduction}

In 1928 Dr. Fred Griffith recorded in his pathology report that the two kinds of colonies, rough and smooth colonies of S. pneumonia are seen in microbiological solid growth media (e.g. blood agar plate). Until now these colonies have not been appropriately analyzed. However, such morphological variations (opaque and transparent) of bacterial colonies are also true for several diplococcic Gram-positive pathogens including S. mutans (dental) and S. mitis. Significantly, these diplococcic Gram-positive pathogens have low G-C content $(<40 \%)$ but they differ in their pathogenesis. In support of our statement, it has been known that the virulence associated proteins CBP's, CbpA, PspA and PepA are found only in $S$. pneumoniae except $S$. mitis is phylogenetically related to $S$. pneumonia $[1,2]$. Above all, this pathogen is becoming increasingly resistant to modern medicines, antibiotics and surface antigen based polyvalent vaccines and therefore, the disease "bacterial pneumonia" has created a new concern. In order to save children from pneumonia, alternative therapies which affect bacterial genetic ability used to communicate among the members of their own community are urgently needed. Signal based communication is also controlling their gene transfer ability.

A low-calorie sugar alcohol, xylitol, has long been promoted as a preventive agent for dental caries primarily caused by Streptococcus mutans [3-5]. Based on our previous work, we believe that, in addition to dental caries, an appropriately developed preventive use of xylitol may also reduce the incidence of community acquired pneumonia, blood stream infections, meningitis, sinusitis and acute otitis media in children [2-9]. The causative bacterium of these diseases is primarily the Gram-positive diplococcic S. pneumoniae which does not differ from the viridans group streptococci, specifically $S$. mitis, in morphology and metabolism [2-9]. Importantly, these diplococcic pathogens also carry loci for the partial utilization of xylitol $[2,10]$. Studies on S. pneumoniae have shown that an exposure to xylitol causes alteration of surface structure(s) and decreases the bacterial adherence to both biotic and abiotic surfaces $[7,11]$. This observation is further supported by more recent work showing that the xylitol damages the surface ultrastructure of Pneumococci when compared to the effect of fructose or sucrose $[7,12]$. We have also observed that the $S$. mitis shows neither lysis nor any bactericidal effect while they are grown to saturation in the presence of xylitol. Our analyses by the Raman spectroscopy have previously confirmed formation of a xylitol-derivative that stably affects the cell division of the parents (an irreversible genetic effect) [2]. We report here that xylitol-treated $S$. mitis becomes sensitive to the combination of antibiotics, colistin (polymyxin B or E) and nalidixic acid, resembling the behavior of Gram-negative E. coli K-12. The major difference of these Gram-positive and Gram-negative bacteria is their cell wall thickness [13].

\section{Material and Methods}

A genetically pure culture of $S$. mitis in brain heart infusion (BHI) was grown overnight in a $\mathrm{CO}_{2}$ jar. After approximately 24 hours, the culture was diluted to $10^{-4}$ and $10^{-5}$ in $\mathrm{BHI}$ and allowed to grow for 2 to 3 hours. Then, $0.1 \mathrm{ml}$ from each dilution was spread onto trypticase soy agar (TSA), and TSA containing colistin and nalidixic Acid (CNA) plates, and incubated for 24 to 72 hours under three different conditions: a) $37^{\circ} \mathrm{C}$ without $\mathrm{CO}_{2}$, b) $37^{\circ} \mathrm{C}$ with $\mathrm{CO}_{2}$ and c) $25^{\circ} \mathrm{C}$ (room temperature). The titer was the same under all three conditions. We have used 2 different strains obtained from ATCC collection: ATCC 6249 (closely related to S. pneumoniae) and S. mutans: ATCC 25175 (dental pathogen). Interestingly, S. mutans shows a growth problem at a $\mathrm{pH}$ higher than 7 (unpublished data).

To establish the growth curve for S. mitis, all overnight cultures in BHI medium were diluted 1:50 by adding $0.1 \mathrm{ml}$ of each culture to two separate tubes containing $4.0 \mathrm{ml}$ of fresh $\mathrm{BHI}$ and allowed to grow at $37^{\circ} \mathrm{C}$ in a water bath shaker. Samples were withdrawn at five different

*Corresponding author: Sunil Palchaudhuri, Department of Immunology and Microbiology, Wayne State University, School of Medicine, Detroit, MI, USA, Tel: 313-577-1313; Email: spalchau@med.wayne.edu

Received November 25, 2013; Accepted December 10, 2013; Published December 12, 2013

Citation: Czarnecki G, Dissanayake P, Palchaudhuri S (2013) Gram-Positive S. mitis Become Sensitive to Colistin and Nalidixic Acid when Grown in Xylitol J Mol Genet Med 7: 92 doi: 10.4172/1747-0862.1000092

Copyright: @ 2013 Czarnecki G, et al. This is an open-access article distributed under the terms of the Creative Commons Attribution License, which permits unrestricted use, distribution, and reproduction in any medium, provided the original author and source are credited 
time intervals and each sample diluted to $10^{-4}$ in phosphate buffered saline. After dilution, $0.1 \mathrm{ml}$ of each culture was spread onto the TSA plates and incubated at $37^{\circ} \mathrm{C}$. The bacterial titer was determined on a solid agar media by counting the colony forming units (CFU). The growth curve for the $S$. mitis in $2 \%$ xylitol was established by preparing an overnight culture in BHI and incubating in a candle jar for 24 hours. This culture was diluted 100 -fold. Then, $1 \mathrm{ml}$ was transferred to two separate tubes. The first tube was a control to which nothing was added. To the second tube, $0.1 \mathrm{ml}$ of a $20 \%$ xylitol (stock solution) was added. Both tubes were incubated in a water bath at $37^{\circ} \mathrm{C}$ with shaking. Samples were withdrawn at different time intervals. At each interval, 0.1 $\mathrm{ml}$ was diluted to $10^{-3}$ or $10^{-4}$ in phosphate buffered saline and spread onto the TSA and CNA plates. The plates were incubated at $37^{\circ} \mathrm{C}$ and the colonies were counted after 24 hours. The logarithmic growth of the bacteria was monitored by counting the colonies to establish the number of colony forming units in different rich agar (solid) media. There was no hemolysis or lysis of intact bacteria in blood agar media. The MIC procedures to examine the alteration of the antibiotic sensitivity patterns induced by the presence of xylitol in the S. mitis growth media were also modified [14].

\section{Scanning electron microscopy (SEM)}

S. mitis was grown in $\mathrm{BHI}$ containing $2 \%$ xylitol for overnight at $37^{\circ} \mathrm{C}$, and fixed with $2.5 \%$ glutaraldehyde. The sample was washed serially with $25 \%$ absolute ethanol. After shadowing with gold for 5 seconds, the sample was visualized under JEOL JSM-7600F at $15 \mathrm{kV}$ [15].

\section{Results}

\section{Altered growth pattern of $S$. mitis}

To further confirm our observations, we followed the growth pattern of the S. mitis in rich broth (TSB) with and without xylitol. Our data (Figure 1) demonstrated that they grew much slower in the presence of xylitol. Their generation time was prolonged, causing a 50 -fold reduction of the final titer compared with the control grown similarly but without xylitol. Furthermore, unlike the growth pattern of the Gram-negative E. coli, the diplococcic viridans group streptococci (S. mitis) did not show any lag period but immediately initiated growth when their overnight cultures were diluted in the fresh nutrient broth. In the presence of xylitol (2\%), this growth rate declined but continued at a much slower rate to the titer of $10^{7} / \mathrm{ml}$ while the titer of the control culture ( $0 \%$ xylitol) nearly reached a titer of $10 \% / \mathrm{ml}$ during the same period of growth. A marked decline in the growth rate occurred after one hour in $2 \%$ xylitol. On the basis of our growth curve as presented

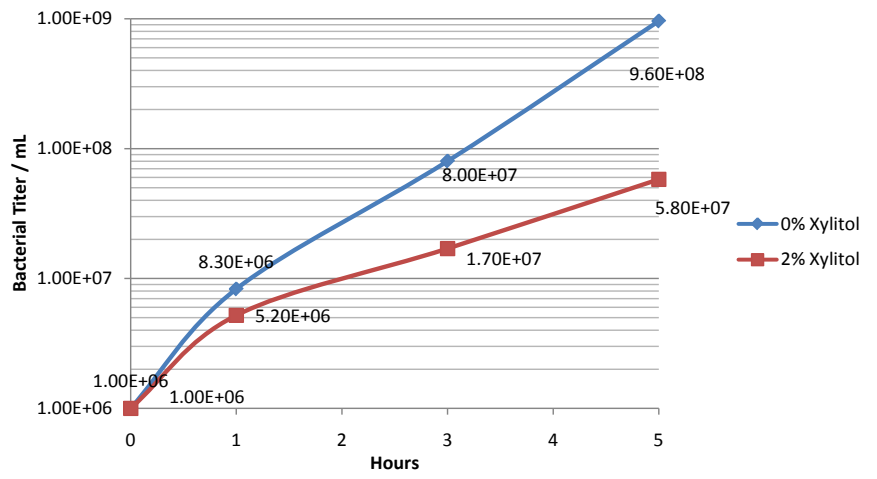

Figure 1: Growth curves of viridans group streptococci (S. mitis) in $0 \%$ xylitol and $2 \%$ xylitol. in Figure 1, we cannot compute the generation time but after 5 hours of growth, the xylitol-exposed $S$. mitis were unable to reach the same titer as the control $\left(10^{7} / \mathrm{ml}\right.$ vs. $\left.10^{9} / \mathrm{ml}\right)$.

The maximum effect was observed at the $2 \%$ xylitol. At concentrations of xylitol above $2 \%$, the rate of growth did not decrease further but it reached a plateau. Our data confirms that xylitol can influence the $S$. mitis metabolism even in the rich broth containing dextrose. This finding is in agreement with the thesis work of Dr. Tapiainen [7]. To minimize the population of dead or old cells, we have diluted our overnight culture 10,000-fold and examined the morphological homogeneity of the diplococcic population immediately after dilution in fresh broth [2]. The overnight culture was also grown in an anaerobic jar with gentle shaking but the final titer did not differ even if they were grown aerobically.

\section{Xylitol-induced alteration of antibiotic sensitivity}

We grew the $S$. mitis in rich broth with varying concentrations of xylitol $(0 \%$ to $6 \%)$ from early log-phase to saturation $\left(10^{4} / \mathrm{ml}\right.$ to $\left.10^{9} / \mathrm{ml}\right)$ (Figure 1). The xylitol-exposed bacteria were then spread onto three different growth media: blood agar, TSA, and TSA containing antibiotics colistin and nalidixic acid (CNA). After 24 hours of incubation at $37^{\circ} \mathrm{C}$ with and without $\mathrm{CO}_{2}$, an approximately equal number of colonies grew on all three media; but, colonies on CNA were much smaller in size compared to the colonies grown on blood agar and TSA media that contained no antibiotics. When we transferred these small colonies (Lederberg's technique) to another fresh CNA plate containing 2\% xylitol, the transferred colonies showed a serious inhibitory effect and became visible only as micro-colonies after 48 hours of incubation at $37^{\circ} \mathrm{C}$. This data leads to a conclusion that these micro-colonies are due to the complete inhibition of cell multiplication (or cell division), or both, and the partial utilization of xylitol has made them sensitive to the combined action of antibiotics, colistin and the nalidixic acid $(1 \mu \mathrm{g} / \mathrm{ml}$ each). The colistin and nalidixic acid could reach their targets located in the membrane of $S$. mitis after their growth for 5-7 generations in $2 \%$ of xylitol. However, such inhibitory effect was much less when these antibiotics were added separately. To confirm this observation, an agar dilution test was performed after the required modification of the protocol of Baker et al. as reported below [14].

\section{Measurement of antibiotic sensitivity by MIC}

In the agar dilution test, the bacteria were diluted in Muller-Hinton broth to reach an approximately titer of $10^{6} \mathrm{CFU}$ and spread on the surface of the TSA medium [2]. In our experiment these TSA plates contained varying concentrations of antibiotics $(0 \%$ to $100 \mu \mathrm{g} / \mathrm{ml})$, colistin and nalidixic acid separately or together. However, the stock solutions of these two antibiotics were prepared in sterile distilled water and filtered through Millipore filter discs, with a pore size of 2 $\mu \mathrm{m}$. After autoclaving, the solid agar TSA medium was cooled to $47^{\circ} \mathrm{C}$ by leaving them in a water bath for 45 minutes and then different concentrations of antibiotics were added (Table 1). We have also recorded that the commercially available CNA media containing these antibiotics lose their potency after autoclaving but still Gram negative E. coli $\mathrm{K}-12$, S. typhimurium and numerous air-borne bacteria do not show any growth during their storage at room temperature or even after 48 hours of incubation at $37^{\circ} \mathrm{C}$ with or without $\mathrm{CO}_{2}$. In support of this observation, the Gram-positive $S$. mitis have conditionally become sensitive to antibiotics as evidenced by the formation of micro-colonies in the presence of colistin (polymixin B or E) and nalidixic acid when used in combination (Table 1 ). 
Citation: Czarnecki G, Dissanayake P, Palchaudhuri S (2013) Gram-Positive S. Mitis Become Sensitive to Colistin and Nalidixic Acid when Grown in Xylitol J Mol Genet Med 7: 92 doi: 10.4172/1747-0862.1000092

Page 3 of 4

Comparison of xylitol-grown $S$. mitis by optical microscopy and SEM

At all concentrations of xylitol, the bacteria are similarly grown to saturation (5-hours) with and without shaking. When these xylitol- exposed populations are visualized by the century old Gram-staining technique, the majority of the bacteria grow in chains and display a combination of color both purple and pink and a very few purple diplococcic individuals (Figure 2B). Obviously, the pink segment of the

\begin{tabular}{|c|c|c|c|c|c|c|}
\hline \multirow[t]{2}{*}{ Bacteria grown with and without $2 \%$ xylitol } & \multirow[t]{2}{*}{ *TSA } & \multicolumn{5}{|c|}{ TSA with Colistin and Nalidixic acid } \\
\hline & & $1 \mu \mathrm{g} / \mathrm{ml}$ & $2 \mu \mathrm{g} / \mathrm{ml}$ & $5 \mu \mathrm{g} / \mathrm{ml}$ & $10 \mu \mathrm{g} / \mathrm{ml}$ & $15 \mu \mathrm{g} / \mathrm{ml}$ \\
\hline S. mitis without xylitol & + & + & + & + & + & $+/-$ \\
\hline S. mitis in $2 \%$ xylitol for $2.5 \mathrm{Hr} * *$ & + & + & - & - & - & - \\
\hline S. mutans without xylitol & + & + & - & - & - & - \\
\hline S. mutans in $2 \%$ xylitol & + & - & - & - & - & - \\
\hline E. coli K-12 without xylitol & + & - & - & - & - & - \\
\hline E. coli $\mathrm{K}-12$ in $2 \%$ xylitol & + & - & - & - & - & - \\
\hline
\end{tabular}

"+" means growth, "-" means no growth, "+/-" means very little growth

*Plating was repeated on Mueller-Hinton Agar as well as TSA

** $S$. mitis when grown beyond 3 hours in the presence of xylitol, starts showing a significant decrease in their titer as measured by their colony forming units (cfu). Therefore, their antibiotic sensitivity pattern was compared after 2.5 hours of growth at $37^{\circ} \mathrm{C}$.

Table 1: Antibiotic sensitivity of S. mitis in $2 \%$ xylitol as measured by agar dilution test.

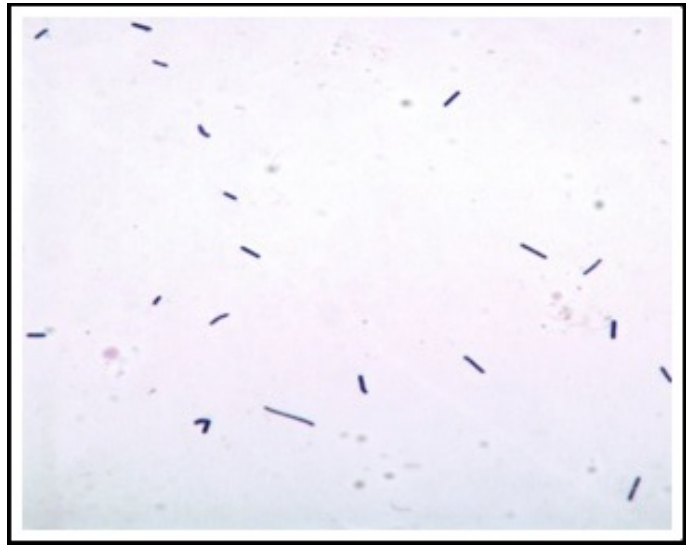

A

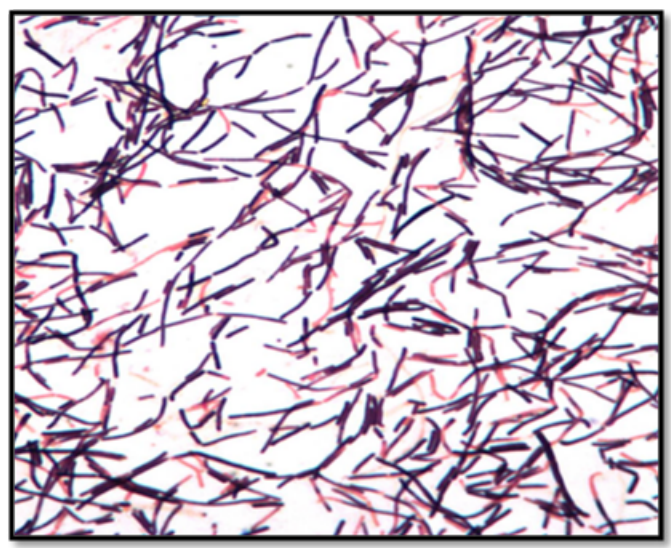

$\mathrm{B}$

Figures 2A and B: Gram-stain of S. mitis control (A) and (B) exposed to $2 \%$ xylitol. Figure $2 \mathrm{~B}$ shows $S$. mitis in chains with a combination of colors. They represent two different growth phases and therefore their titer differs.

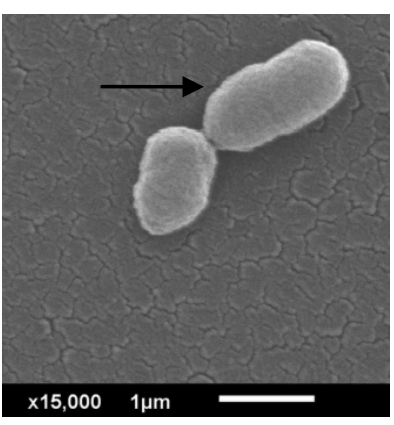

A

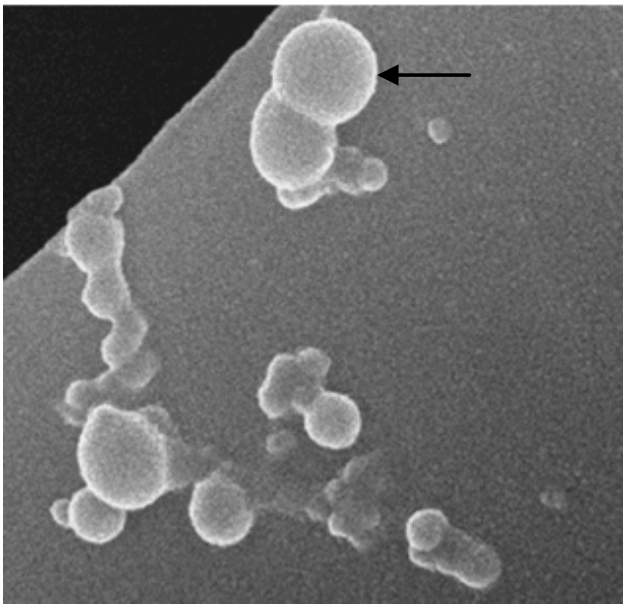

B

Figures 3A and 3B: Scanning electron micrograph of S. mitis in $2 \%$ xylitol. There is heterogeneity of sizes after exposing to xylitol . Partially damaged bacteria are larger, still smaller than the normal size. Arrow shows the normal size $S$. mitis $(\sim 1.8 \mu \mathrm{m})$. 
same purple chain appears very thin and fragile. Failure of crystal violet to stably interact with the xylitol-grown Gram-positive bacteria leads to a conclusion that the cell wall thickness has been affected. SEM analysis of the same population exposed to $2 \%$ xylitol confirms their presence in a long chain but in heterogeneity of sizes (Figure 3B). Figure 3A shows bacterial control without xylitol. In Figure 3B there are a few larger bacteria in the same chain. These are probably the live ones which have been partially affected by xylitol but are still capable of forming colonies [12]. These colonies become sensitive to nalidixic acid and colistin when used in combination (Table 1). In the same micrograph there is a presence of normal size diplococcic parent (about $1.8 \mu \mathrm{m}$ ). The optical density values at $580 \mu \mathrm{m}$ showed no difference between the control and the $2 \%$ xylitol-exposed population. What is more, the optical density values do not show any difference indicating no lysis.

\section{Discussion}

Unlike penicillin or lysozyme, xylitol does not lyse the bacterial cell wall, but bacterial multiplication is gradually affected. Evidently, the cell division mechanism of diplococcic bacteria belonging to both Gram-positive and Gram-negative needs further exploration. It is well-established that the duplication and subsequent error-free segregation of chromosomes into daughter cells requires an effective association with cell wall/membrane complex (cytokinesis) [16]. Our data indicates that the stable presence of xylitol derivative, formed by partial metabolism of xylitol, in S. mitis interferes in their normal biosynthesis and therefore their generation time is prolonged (Figure 1). The optical densities of the bacterial cultures with and without xylitol do not differ very much but the xylitol-exposed diplococci grow in long chains regardless of their chain lengths. Obviously, Griffith's rough and smooth colonies can be explained by revisiting his old data when we accept that this pathogen grows in chains (latent or stationary phase) and they will produce a single colony (rough) instead of separate colonies (Palchaudhuri et al, Ms. in preparation). We have also observed by the Gram staining technique that the majority of the xylitol-exposed diplococci are stably present in chains with heterogeneity of color within the same chain (Figure 2B). SEM analysis of the same population exposed to $2 \%$ xylitol confirms their presence in a long chain and shows heterogeneity of sizes (Figure 3B).The pink is indicative of death or reduction of cell wall thickness resembling the staining pattern of Gram negative E. coli. Consequently, those that survive the exposure to xylitol become sensitive to the combination of the antibiotics colistin and nalidixic acid. We speculate that the reduction of cell wall thickness caused by the accumulation of xylitol derivative may interfere in their normal cell division.

\section{Acknowledgement}

We would like to thank Dr. A. Palchaudhuri for the partial financial support and her knowledge in clinical microbiology.

\section{References}

1. Johnston C, Hinds J, Smith A, van der Linden M, Van Eldere J, et al. (2010) Detection of large numbers of pneumococcal virulence genes in streptococci of the mitis group. J Clin Microbiol 48: 2762-2769.

2. Palchaudhuri S, Rehse SJ, Hamasha K, Syed T, Kurtovic E, et al. (2011) Raman spectroscopy of xylitol uptake and metabolism in Gram-positive and Gram-negative bacteria. Appl Environ Microbiol 77: 131-137.

3. Trahan $L$ (1995) Xylitol: a review of its action on mutans streptococci and denta plaque--its clinical significance. Int Dent J 45: 77-92.

4. Loesche WJ (1985) The rationale for caries prevention through the use of sugar substitutes. Int Dent J 35: 1-8.

5. Kontiokari T, Uhari M, Koskela M (1998) Antiadhesive effects of xylitol on otopathogenic bacteria. J Antimicrob Chemother 41: 563-565.

6. Uhari M, Kontiokari T, Koskela M, Niemelä M (1996) Xylitol chewing gum in prevention of acute otitis media: double blind randomised trial. BMJ 313: 11801184

7. Tapiainen T (2002) Microbiological effects and Clinical use of Xylitol in preventing Acute Otitis Media. (PhD thesis). University of Oulu.

8. Salyers A, Whitt DD (2002) Bacterial pathogenesis: A Molecular Approach ASM Press, Washington, D.C.

9. Gladwin M, Trattler B (2011) Clinical Microbiology. 5th ed. Med Master, Miami.

10. Waldor MK, Friedman DI, Adhya SL (2005) Phages, their role in pathology and biotechnology. ASM Press, Washington, D.C.

11. Ghannoum M, O'Toole GA (2004) Microbial biofilms. ASM Press, D.C

12. Tapiainen T, Sormunen R, Kaijalainen T, Kontiokari T, Ikäheimo I, et al. (2004) Ultrastructure of Streptococcus pneumoniae after exposure to xylitol. J Antimicrob Chemother 54: 225-228.

13. Johnson A (2005) Antimicrobial Agents. J Antimicrob. Chemother 58: 231.

14. Baker CN, Stocker SA, Culver DH, Thornsberry C (1991) Comparison of the Test to agar dilution, broth microdilution, and agar diffusion susceptibility testing techniques by using a special challenge set of bacteria. J Clin Microbio 29: $533-538$.

15. Palchaudhuri S, Dissanyake P, Palchaudhuri A (2013) Blocking the two component signal transduction pathway of diplococcic Gram-positive pathogens by xylitol cloud as visualized by SEM. Intl. Conference in India, 43-44.

16. Nanninga N (2001) Cytokinesis in prokaryotes and eukaryotes: common principles and different solutions. Microbiol Mol Biol Rev 65: 319-333. 